\title{
Localisation of Subdural EEG Electrode Bundles in an Interactive Volume Rendering Framework
}

\author{
H.J. Noordmans ${ }^{1}$, C.W.M. van Veelen ${ }^{2}$, and M. A. Viergever ${ }^{1}$ \\ ${ }^{1}$ Image Sciences Institute E.01.334 \\ ${ }^{2}$ Dept. Neurosurgery G03.124 \\ University Hospital Utrecht, P.O. Box 85500, 3508 GA Utrecht, The Netherlands \\ herke@isi.uu.nl \\ http://www.isi.uu.nl.
}

\begin{abstract}
When the focus of epilepsy is so deep that skin EEG electrodes do not give enough accuracy in calculating the position of the focus, it may be decided to surgically implant EEG electrodes inside the patient's head. To localise these electrodes, a high resolution 3D CT scan is made of the patient's head. As manual tracking of the electrodes slice by slice is tedious and erroneous, a virtual reality environment has been created to give the radiologist a view from inside patient's skull. With the help of a high quality but fast volume renderer, the radiologist can get an overview of the electrode bundles and can interactively characterise the bundle of interest. For the localisation of the lead markers, we compared manual placement, centre of gravity and Gaussian image matching. For the interpolation, we compared line and NURBS interpolation with the optional restriction of equal segment size or zero curvature at the end of the bundle. It appeared that the electrodes could be characterised with high accuracy, that manual positioning equally performed as centre of gravity methods, and that NURBS interpolation with equal segment size outperformed line interpolation and NURBS interpolation without the equal segment restriction.
\end{abstract}

\section{Introduction}

For patients with local epilepsy, it is sometimes difficult to determine the exact location of the focus of epilepsy. The first option is to put EEG electrodes on the skin of the patient's head but when the focus lies deeper in the brain, the accuracy in determining the focus ${ }^{1}$ is often limited. In such cases, it can be decided to operatively implant EEG electrodes inside the patient's head (2,5, Fig. 1). Then often two bundles, called depth bundles, are inserted with a hollow needle into the head of the hippocampi. Other bundles are inserted through a bore hole and shifted over the brain cortex, the subdural bundles. The electrodes are embedded in plastic and make contact with the surrounding tissue at locations where isolation has been removed. A depth bundle contains six contacts, a subdural bundle seven. The subdural bundles have three lead markers to localise the bundles in an X-ray scan; the depth bundles do not have these markers. 


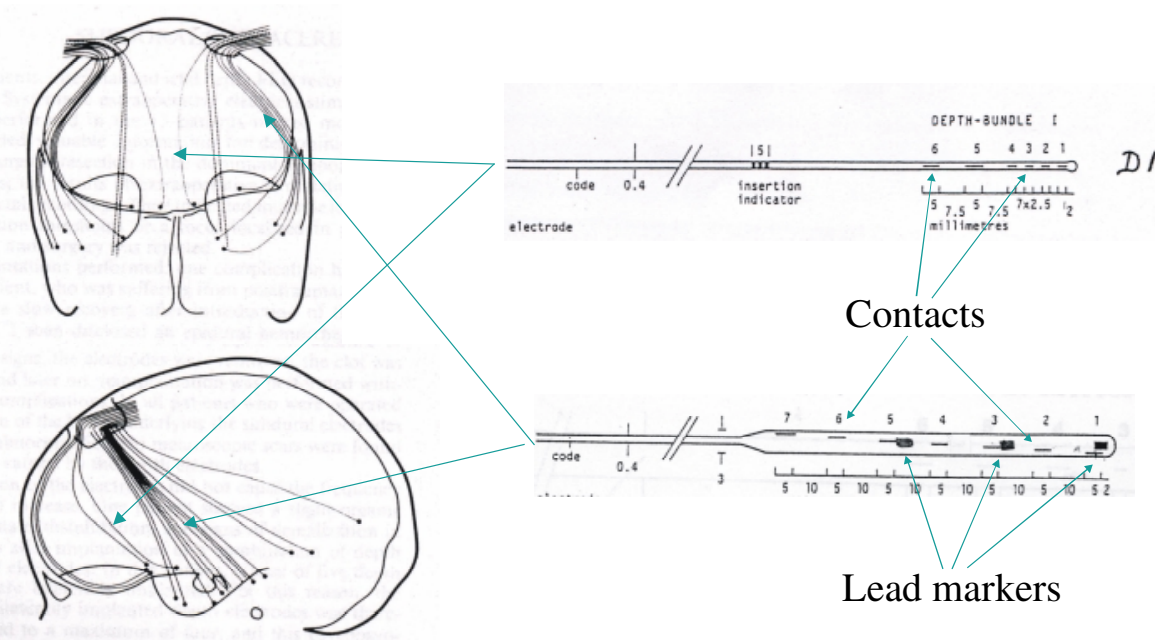

Fig. 1. Technical drawing of the implanted EEG electrode bundles.

Apart from the surgical procedure and the characterisation of the depth bundles, the biggest problem is to recognise the subdural bundles on an X-ray scan. Although a high resolution 3D X-ray scan is made of the patient's head and the individual lead markers can be distinguished from each other, it is often difficult to determine from X-ray slices which lead markers belong to the same bundle 2 (Fig. 2). Problems are that bundles are inserted from the same bore hole and therefore often cross in the patient's head. Also, bundles are sometimes so close to the skull that they seem to vanish.

We try to solve these problems by giving the user a 3D view from the inside of the patient's skull (Fig. 3). Thanks to the overview, the user has less difficulty to recognise the individual electrode bundles and to interpret the information from the scanned slices. If the rendering quality is high enough, it is also easier to visually connect separate parts of the electrode bundles than by looking at individual slices.

We use volume rendering to generate the 3D view. We chose for that option instead of using fast graphics on specialised hardware (e.g. OpenGL), as we did not accept possible errors introduced by converting the volume data to graphical surface patches. This erroneous 'segmentation' step is avoided in volume rendering, by which the image quality is higher and the user can visually check his segmentation results with the original data.

For the problem of localising the electrode bundles, we developed a 3D interactive system consisting of a high qualitative, but fast volume renderer combined with interactive tools enabling the user to accurately characterise surgically implanted EEG electrodes. The user indicates a subdural bundle by placing artificial blobs in the neighbourhood of lead markers. The computer refines the positions of the artificial blobs and fits a curve through the three positions. The final curve is showed, eventually together with the interpolated EEG contacts. The user then accepts the result or adapts his initial guess. 


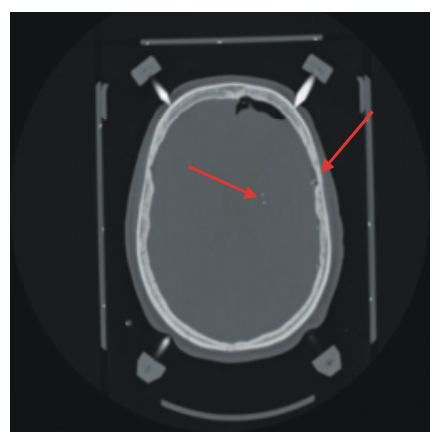

Fig. 2. CT slice showing the two depth electrode bundles and three subdural EEG bundles next to the skull.

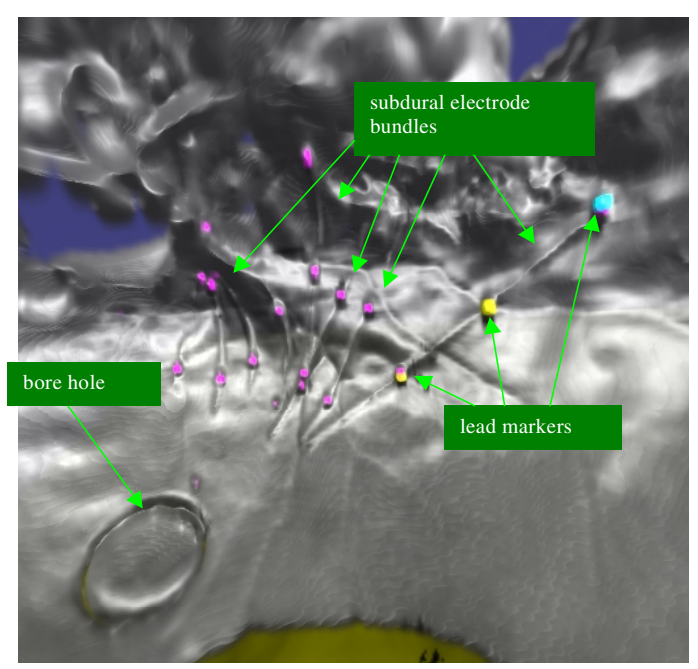

Fig. 3. Volume rendering of EEG electrode bundles implanted inside the skull of a patient with epilepsy. Through a bore hole in the skull, the electrode bundles are pushed over the cortex. Each bundle contains 7 contacts (not visible) and three lead markers (purple blobs). The user moves one magenta and two yellow artificial blobs to the lead markers to indicate which bundle he/she wants to characterise.

A 3D CT scan is made of the head of the patient. Matrix size $512 \times 512 \times 90$, FOV $300 \mathrm{~mm}$, slice thickness 1.5mm (Philips Tomoscan SR7000). The data is read on a SGI workstation with four R10000 processors running at $194 \mathrm{MHz}$, and $1 \mathrm{~GB}$ of memory. After cropping the data to remove the stereotactic frame mounted on the head (remaining matrix approximately 300x400x90), the data is thresholded to segment the skull, the electrode bundles and the lead markers. A second threshold is used to segment the more intense lead markers.

The original data set and the two segmentations are combined in a volume rendering to show the user the original data. We use Phong shading to enhance surface detail 5, a semi-transparent skull to illuminate the inner side of the skull, and a table with a shadow as a projection of the data set. To get a fast initial visual response, the renderer uses optimisation schemes like adaptive ray termination, template based viewing, adaptive progressive refinement, presence sampling, blur prevention, local volume update, and view movement 3.7. The result is a first update within a second and a final update in 10 seconds.

An overview of the system is given in Fig. 4. In the window with name para_0, the user gets an overview of the entire data set (parallel projection). The windows persp_0 and persp_1 give the user a look inside the head of the patient (perspective projection). The subdural bundles are visible as ridges on the skull, the depth bundles appear as antennas in the emptied skull. The magenta dots denote the lead markers. The window plane_0 shows an arbitrarily formatted slice. By dragging the mouse inside a parallel, perspective view or plane, the user can interactively change the view 
position or direction. The user can move the perspective viewpoint by using the step buttons in the perspective view control panel.

After selecting the appropriate viewpoints, the measurement starts by creating three artificial blobs: A cyan blob to denote the lead marker at the end of an electrode bundle, and two yellow blobs for the other two lead markers. The user moves the blobs in the neighbourhood of the desired lead markers by selecting them through any of the view or planes, and dragging them with the mouse. The computer matches the positions of the blobs to the real marker positions and fits a curve to the three positions. The final curve and the calculated contacts are combined with the original volume rendering to let the user qualitatively validate the result.

The artificial objects are visualised as voxel objects instead of graphics as a hybrid volume renderer would be more difficult to implement, computational complex, and would easily create artefacts at subvoxel scale (e.g. does a graphic intersect the data at the visual correct position?). We therefore voxelize all artificial objects and draw them in a geometry volume. An additional label volume specifies their colour. An additional advantage of voxelized objects is that is relatively easy to identify the artificial objects the user has selected through a view. Starting from the position where the user presses a mouse button, a search is performed in the view direction to find the first artificial object. This is implemented by tracing rays through a so-called identification volume, which is derived from the voxel representations of the artificial objects.

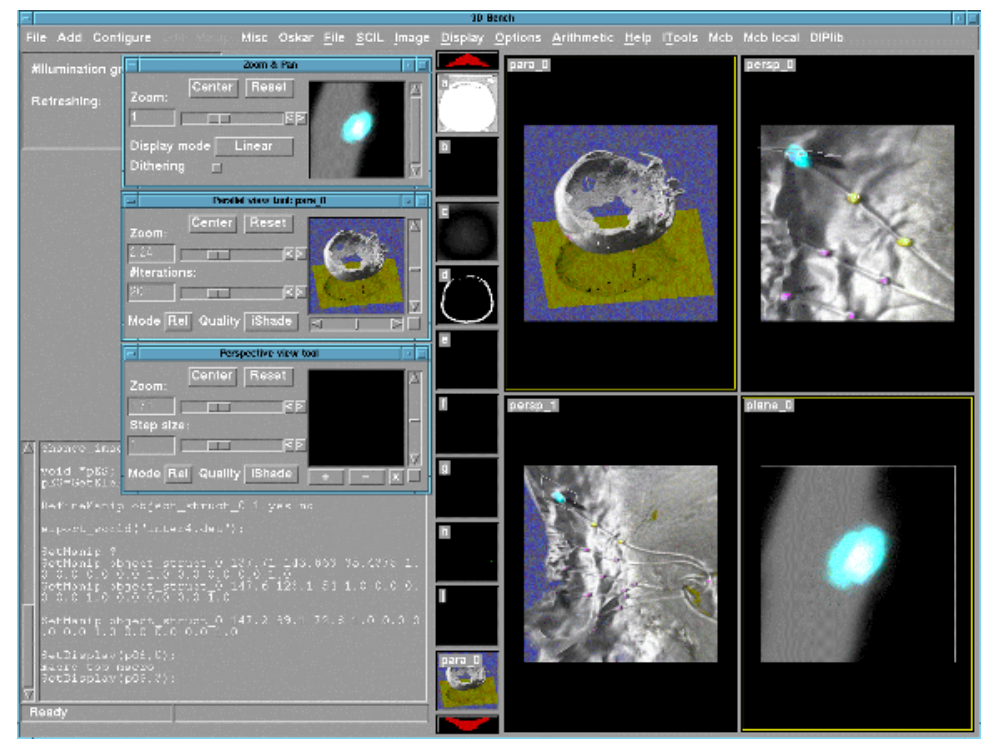

Fig. 4. Overview of the EEG bundle localisation system. Windows: para_0, fast overview using parallel projection; persp_0, persp_1, two perspective projections from inside; plane_0, arbitrarily orientable slice. The windows zoom tool, plane tool and perspective view tool are display, respectively view control panels. The left-bottom panel shows the command line Cinterpreter. 


\subsection{Localisation Techniques}

To improve the accuracy and robustness in localising the lead markers, the computer refines the position indicated by the user. We looked at three refinement techniques:

Centre of gravity. The final lead marker position is calculated by averaging over the marker co-ordinates $\mathbf{X}$ in a local environment around the user-specified region $V$,

$$
\mathbf{m}=\frac{\int_{d} \mathbf{x} f(I(\mathbf{x})>0) d \mathbf{x}}{\int_{d} f(I(\mathbf{x})>0) d \mathbf{x}},
$$

with $I(\mathbf{x})$ the image values and $f()$ the indicator function.

Intensity weighted centre of gravity. The image co-ordinates are weighted by the image intensity:

$$
\mathbf{m}=\frac{\int_{d} \mathbf{x} I(\mathbf{x}) d \mathbf{x}}{\int_{d} I(\mathbf{x}) d \mathbf{x}} .
$$

Weighted image matching with a Gaussian profile 8. Starting from the initial guess, the computer changes a parameter vector $\mathbf{p}$ to optimise the match between the Gaussian intensity model and image data:

$$
\varepsilon=\frac{\int_{d}(I(\mathbf{x})-F(\mathbf{p} ; \mathbf{x}))^{2} G(\mathbf{p} ; \mathbf{x}) d \mathbf{x}}{\int_{d} G(\mathbf{p} ; \mathbf{x}) d \mathbf{x}},
$$

with parameter vector $\mathbf{p}=(\mathbf{x}, a, \sigma)$, Gaussian profile $F(\mathbf{p} ; \mathbf{x})=a e^{-\frac{|\mathbf{x}|^{2}}{2 \sigma^{2}}}$, and weight function $G(\mathbf{p} ; \mathbf{x})=e^{-\frac{|\mathbf{x}|^{2}}{2 \rho^{2} \sigma^{2}}}$.

\subsection{Interpolating Curve}

To determine the positions of the EEG contacts from the lead marker positions, we investigated two techniques:

Line interpolation. Fig. 5a. Draw two lines, one from the first to second marker, and one from the second to third marker.

Second degree NURBS interpolation 4. Fig. 5b. Generally, none of the $\mathrm{n}$ derivatives of the NURBS is specified. As the electrode bundle has a free end, it is sensible to specify a zero $2^{\text {nd }}$ derivative at that end. We also know that the arclength between marker 1 and 2 and the arclength between marker 2 and 3 should be $30 \mathrm{~mm}$, thus equal. As the knot vector contains one free parameter, we can change it such to make the calculated arclengths equal. We therefore looked at four interpolation options: (1) free arclengths + no zero curvature restriction, (2) equal arclengths + no zero curvature restriction, (3) free arclengths + zero curvature restriction, and (4) equal arclengths + zero curvature restriction. 


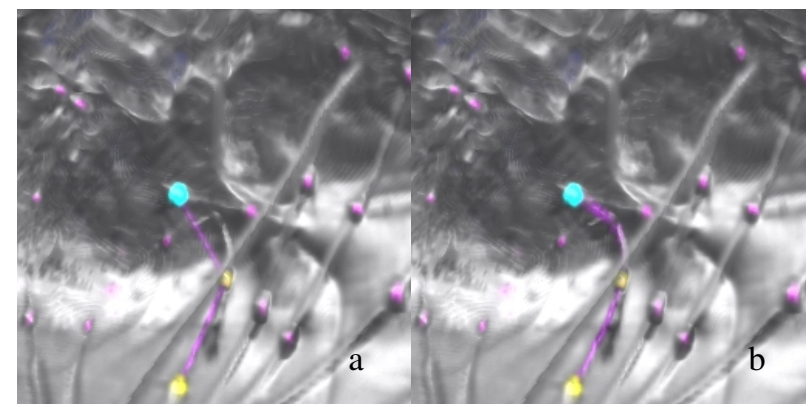

Fig. 5. Line interpolation (a) versus curve interpolation (b).

\section{Evaluation}

We tested the system on four patients with epilepsy. The numbers of implanted subdural bundles were 3, 3, 7, and 13; 26 in total. We evaluated the measurement qualitatively by comparing the interpolating curve with the original electrode bundle. We evaluated the measurement quantitatively by comparing the measured arclength between the first and second marker, and the arclength between the second and third marker to the value of $30 \mathrm{~mm}$ expected from the technical specifications.

\section{Results}

Qualitative. The complete procedure is illustrated in Fig. 6. At the start of the procedure, where no EEG bundles are characterised yet, the user has a good view on the inside of the skull and the imprints of the EEG bundles on the CT scan (a). After localising the subdural EEG bundles, the interpolating NURBS are shown (green) together with the interpolated contact positions (red) (b). After the depth electrode is tracked, its measurements are reconstructed as red rods (c). Finally, the results are merged with a CT-registered 3D T1 MRI scan (d).

Quantitative validation. After manual placement, the computer refines the location of the artificial blobs by one of the three techniques mentioned above. Then the computer fits a line or curve and measures the arclengths. The arclengths after each measurement are summarised in Table 1. Each time, the first row shows the mean and standard deviation for the segment between the end marker and the second marker. The second row shows the mean and standard deviation for the segment between the second and third marker.

We saw that, when looking qualitatively at the results, line interpolation gave larger errors than curve interpolations (Fig. 5). Regarding curve interpolation, the largest errors occurred when the end segment was strongly bent and the interpolating curve had a slightly lower curvature (images not shown). The calculated contact was then estimated to be 1 or $2 \mathrm{~mm}$ from the real contact position. 


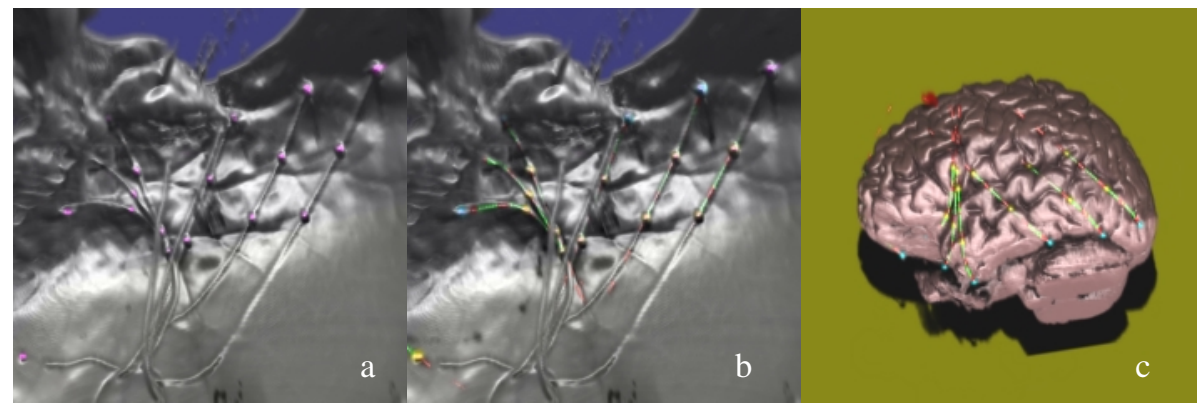

Fig. 6. Overview of the electrode characterisation process: a Initial view showing the electrode bundles and lead markers (purple). b Result after bundle characterisation: Interpolated NURBS splines (green) and contact positions (red). c Bundles visualised on segmented brain from a registered 3D T1 MRI scan.

Table 1. Mean and standard deviation of measured segment lengths ( $\mathrm{mm})$.

\begin{tabular}{|c|c|c|c|c|c|c|c|c|}
\hline & \multicolumn{2}{|c|}{ Manual } & \multicolumn{2}{|c|}{ Centre of gravity } & \multicolumn{2}{|c|}{$\begin{array}{l}\text { Intensity weighted } \\
\text { centre of gravity }\end{array}$} & \multicolumn{2}{|c|}{$\begin{array}{c}\text { Gaussian image } \\
\text { matching }\end{array}$} \\
\hline Line & 28.7 & 1.5 & 28.8 & 1.4 & 28.8 & 1.4 & 28.7 & 1.5 \\
\hline interpolation & 29.9 & 0.9 & 29.9 & 1.0 & 29.9 & 1.0 & 29.9 & 0.6 \\
\hline Curve 1 & 29.1 & 1.3 & 29.2 & 1.2 & 29.1 & 1.2 & 29.1 & 1.3 \\
\hline & 30.3 & 0.8 & 30.3 & 0.9 & 30.3 & 0.8 & 30.3 & 0.6 \\
\hline Curve 2 & 30.2 & 0.7 & 30.2 & 0.7 & 30.2 & 0.7 & 30.2 & 0.5 \\
\hline & 30.2 & 0.7 & 30.2 & 0.7 & 30.2 & 0.7 & 30.2 & 0.5 \\
\hline Curve 3 & 28.9 & 1.4 & 28.9 & 1.3 & 28.9 & 1.3 & 28.9 & 1.4 \\
\hline & 30.4 & 0.8 & 30.4 & 0.8 & 30.4 & 0.8 & 30.4 & 0.6 \\
\hline Curve 4 & 30.2 & 0.7 & 30.2 & 0.7 & 30.2 & 0.7 & 30.1 & 0.5 \\
\hline & 30.2 & 0.7 & 30.2 & 0.7 & 30.2 & 0.7 & 30.1 & 0.5 \\
\hline
\end{tabular}

Fig. 7. Errors in marker localisation when markers are close. a Two lead markers at $2 \mathrm{~mm}$ distance. b Artificial blob after manual adjustment. c Refinement with centre of gravity technique; The second marker disturbs the proper calculation of the centre. $\mathbf{d}$ Refinement after match with Gaussian.

From Table 1, the following conclusions can be drawn: (1) The end segment of an electrode bundle has a significantly shorter line length than the second segment; this means that the end of the bundle is always more bent than the second segment. (2) Manual placement is not significantly less accurate than automatic refinement procedures. This, because the user has the disposal of an interactive slice, which 
he/she can zoom in on the actual marker of interest. The slice enables the user to accurately position the artificial blob over the actual marker. (3) Refining the position by matching the marker with a Gaussian is slightly more accurate than the other techniques. The reason is that in some cases the gravity techniques fail when two markers are so close that the centre is found between the two markers (Fig. 7.). Gaussian image matching has less trouble in such cases, as the weight function effectively reduces the disturbing influences of a neighbouring spot of (4) Imposing zero curvature at the end of the bundle does not significantly increase the accuracy, but does not decrease it either. Imposing equal arclengths does increase the accuracy.

In summary, the best technique would be Gaussian image matching of marker positions followed by NURBS fitting with equal length and zero curvature restriction. Less difficult to implement but still giving good results, would be manual placement followed by NURBS fitting with equal length restriction.

\section{Conclusions}

A practically useful method has been presented to characterise surgically implanted EEG electrode bundles. The volume rendering method is fast and gives high quality feedback to accurately locate the lead markers and interpret the interpolating curve. The results showed that manual placement of artificial objects to denote the marker positions is equally accurate as calculating the position centre of gravity techniques, but less accurate than Gaussian image matching. A NURBS interpolating curve with the restriction of equal segment size outperformed line interpolation and NURBS interpolation without the equal segment restriction.

\section{References}

1. B.J. Roth, D. Ko, I. von Albertini-Carletti, D. Scaffidi and S. Sato Dipole localization in patients with epilepsy using the realistically shaped head model. Electroencephalography and clinical Neurophysiology 102, 1997, 159-166.

2. L.C. Meiners, The role of MR in drug-resistant epilepsy with special emphasis on mesial temporal sclerosis. PhD Thesis University of Utrecht, The Netherlands. Chapter 4 and 12, 1997.

3. K.J. Zuiderveld, A.H.J. Koning, M.A. Viergever, Acceleration of ray-casting using 3D distance transforms. Visualization in Biomedical Computing 1992, Proc. SPIE 1808, 1992, 324-335.

4. L. Piegl, W. Tiller, The NURBS book. Springer Verlag, Berlin, Second Edition, chapter 9.2.2, 1997.

5. C.W.M. van Veelen, R.M. Chr. Debets, A.C. van Huffelen, W. van Emde Boas, C.D. Binnie, B. Chir, W. Storm van Leeuwen, D.N. Velis, A. van Dieren, Combined Use of Subdural and Intracerebral Electrodes in Preoperative Evaluation of Epilepsy, Neurosurgery 26(1), 1990, 93-101.

6. B.T. Phong, Illumination for computer generated pictures. Comm ACM 18(6), 1975, 311 317.

7. H.J. Noordmans, A.W.M. Smeulders, H.T.M. van der Voort, Fast volume render techniques for interactive analysis. The Visual Computer 13, 1997, 345-358.

8. H.J. Noordmans, A.W.M. Smeulders, Detection and characterisation of isolated and overlapping spots, Computer Vision and Image Understanding 70(1), 1998, 23-35. 